\title{
The Impact of Mental Health Education on Reducing Adolescent Suicide A Case Study of Shanghai
}

\author{
Linjuan $\mathrm{Cai}^{1, \dagger}$ Yimiao $\mathrm{Han}^{2,}{ }^{*}, \dagger$ Jiannan $\mathrm{Shi}^{3, \dagger}$
}

\author{
${ }^{1}$ Hubei University of Economics, Hubei Province, China \\ ${ }^{2}$ Chengdu University of Technology, Sichuan Province, China \\ ${ }^{3}$ Zhejiang Normal University, Zhejiang Province, China \\ ${ }^{*}$ Corresponding author. Email: hanyimiao@stu.cdut.edu.cn \\ ${ }^{\dagger}$ These authors contributed equally.
}

\begin{abstract}
Suicide is one of the leading causes of death among adolescents today. Schools in different countries all make great efforts to reach the youth, yet there is no conclusive evidence regarding the effect of mental health programs in China. The purpose of this article is to examine the efficacy of mental health education programs on curbing suicide for primary and secondary schools in Shanghai. Through a longitudinal analysis, we compared the suicide rates of adolescents in the years 2008 and 2012 in two districts of Shanghai respectively, namely Jiading and Hongkou. The data is from surveys conducted under the guidance of Shanghai Municipal Center for Disease Control and Prevention. Results show that after the introduction of policy on mental health education, there was a decline in suicide rates among middle school students in both districts. To conclude, mental health education can benefit adolescents via various forms. However, due to limited data, we cannot compare more successive data. Future research can be done with more adequate data on larger scales.
\end{abstract}

Keywords: Mental health education, Adolescent suicide, Shanghai.

\section{INTRODUCTION}

Over recent decades, adolescent suicide has become a public health issue worldwide. Globally, suicide has been proved to be the second leading cause of death among young people aged 15-29 years for both sexes [1]. Even worse in China, suicide is the leading cause of death among 15-34 years old, especially for vulnerable adolescents [2].

To address the worrying phenomenon, great efforts have been made to understand the contributing factors of adolescent suicide and suicidal behavior, among which mental health issues turn out to be one prominent reason. As a result, various prevention programs have been developed and conducted on campus, aiming to provide adolescent students with mental health education as well as a series of assistance to reduce the suicide rate. However, there is little practice on evaluating the effects of mental health education in China. To examine the efficacy of mental health education programs on curbing suicide for primary and secondary schools, this paper, therefore, will compare the suicide rates of adolescents before and after the introduction of mental health policy for schools in Shanghai through longitudinal analysis.

The article develops itself as follows: in the next section, we draw major definitions from existing literature, and introduce mental health issues as a contributing factor to adolescent suicide based on previous studies. The third section further gives thirteen negative mentalities usually found in adolescents. In the fourth section, we present the background where the Chinese national government and Shanghai municipality have promoted mental health programs on campus. In the fifth and sixth sections, we introduce the research method, give the data source and present our comparative analysis. In the concluding section, we argue that the ongoing Shanghai policy on mental health education has significantly reduced suicide-related behaviors (SRBs) in local adolescents. 


\section{LITERATURE REVIEW}

\subsection{Adolescent Suicide}

On the definition of suicide, scholars mainly use the following two kinds: one is to define suicide as an act of 'consciously' harming one's own body in order to end one's life [3]. Another term suicidality also frequently appears, which refers to all SRBs and thoughts, including completing or attempting suicide (a self-inflicted yet non-fatal act), suicidal ideation (thoughts of harming or killing oneself) or communications [4]. In this paper, we use the latter definition that suicide is manifested by both suicidal behaviors and suicidal ideation.

Since adolescence is the transition period of rapid physical, cognitive and psychosocial growth, adolescents are especially vulnerable to multifaceted risk factors of suicide [5]. According to the World Health Organization, the span of adolescence is between the ages of 10 and 19, so our subjects are students in primary and secondary schools, who basically fall into this age group.

Although it can be concluded from the previous studies that adolescent suicide is determined by multiple factors and the factors are intertwined, scholars like Evans et al. have concluded there is overwhelming evidence that psychopathology is the most significant risk factor for both suicide death and suicide attempts among adolescents [6]. The mental disorders contributing to suicidal ideation and attempts mainly include psych ache, depression, hopelessness, etc. [7]. In China, recent studies have also shown the correlations between adolescent mental health problems and dangerous behaviors [8].

\subsection{Negative Mentalities}

Here we introduce thirteen negative mentalities which can lead to dangerous behaviors.

1. Anaclisis: Many teenagers have been spoiled by their parents since they were young. They rely on their parents for everything, so they lack adaptability to the environment and the ability to respond to emergencies.

2. Egoism: Mainly due to the excessive indulgence of their parents, some teenagers tend to be self-centered and act as if the whole world revolves around them. They always think that they are superior to others and want to take the lead in everything. Once they fail to achieve something, the outcome can be detrimental.

3. Selfishness and narrow-mindedness: Teenagers who are egocentric are more likely to be narrow-minded, selfish, and uneasy about trivial things. Taking personal interests too seriously, they might be overcome by strong jealousy and even take revenge in some extreme cases [9].

4. Inferiority complex: When they really set foot in new surroundings without the support of their parents, adolescents may find their intelligence and abilities not as great as expected. Consequently, pessimism about their future would be their first reaction, which gradually develops into low self-esteem [10].

5. Depression: Depression is a more common psychological disorder in middle school students, which makes middle school students feel unable to cope with external pressure.

6. Withdrawal: The main manifestations include diffidence and the tendency to escape from or avoid situations that may be experienced as emotionally or psychologically challenging [11].

7. Anxiety: Anxiety is a complex and continuous emotional state characterized by worry or tension. When people expect a certain dangerous and painful situation to occur, they usually show response of anxiety, which is a universal adaptive response.

8. Fear: Here fear refers to a strong, irrational fear of a certain object or situation. In fact, such seemingly threatening objects or situations generally do not cause danger or threat.

9. Irritability: Teenagers with this emotion are impulsive, irritable and moody. They often have the urge to do something dangerous and stupid, but regret their behaviors after they calm down.

10. Rebelliousness: When reaching puberty, some middle school students tend to take a non-cooperative and confrontational attitude when interacting with teachers or parents.

11. Jealousy: Jealousy is a very unhealthy mentality in competition. The more teenagers are spoiled, the more difficult it is for them to deal with unsatisfactory academic results.

12. Obsessive-compulsive disorder: with this disorder, a person is obsessed with certain thoughts or feels the need to perform certain routines repeatedly. Students with compulsion problem find it hard to concentrate on study.

13. Learning disabilities: Middle school students are overburdened in study, and the psychological pressure is increasing. Facing fierce competition to enter top ranked schools and get a job [9], teenagers with weak learning abilities may feel deeply that their future is hopeless [12].

\subsection{Mental Health Programs}

To cope with adolescent suicide, mental health is the priority in prevention programs, especially in school, where adolescents are the most accessible [6]. Although preventive programs vary from regions and countries, researchers roughly categorize them into three tiers, namely the universal or primary level, the selected or secondary level, and the indicated or tertiary level. The 
first tier targets all individuals in a given population (e.g., school; classroom) in the aim of preventing particular emotional, behavioral, or academic problems. The second tier, as the name suggests, is comprised of more intensive interventions like personal counselling for those students who do not adequately respond to the first tier. The third tier is characterized by highly individualized and specialized interventions for those students who already have suicide attempt behaviors [13]

Supplemented in some cases with training directed toward teachers and other gatekeepers, the first tier of prevention typically features an educational curriculum that aims to increase students' knowledge and awareness about suicidal behavior and encourage troubled students to seek help. In the meanwhile, screening programs are also conducted to identify youth who are at risk for suicidal behavior. Programs like Signs of Suicide (SOS) have resulted in students' increased knowledge about suicide and more positive attitudes about communicating and seeking help in the United States [6]. The Applied Suicide Intervention Skills Training (ASIST), designed for training school staff and other gatekeepers, has developed into the broadest and most frequently applied program worldwide [14]

Many countries are highly aware of adolescent suicide, and have issued a series of policies to reduce the suicide rate of adolescents. Take the United States as an example. On the one hand, the government has given substantial financial support. Since the 1870 s, the annual research funding has reached 1.7 billion dollars. On the other hand, the government continues to improve prevention and control measures [15]. In 2002, President George W. Bush established the President's New Freedom Commission on Mental Health, which provided some agenda setting: Firstly, reducing stigma by integrating mental health awareness into special and routine education courses in schools and providing a naturalistic environment for teenagers. Additionally, gatekeeper training model is implemented to prevent adolescent suicide [16]. This model involves improving the knowledge, attitude and skills of school staff in order to intervene with suicide properly, providing crisis intervention training and helping students with suicide risk with treatment and counseling. Schools also offered screening of co-occurring mental health and substance use problems.

In 2007, the School Mental Health Act was proposed to provide a large amount of funds for local educational institutions to expand the existing school mental health work through community-family-school partnership. The bipartisan legislation emphasized the public health methods for mental health, including prevention and promotion, positive behavior support and targeted intervention, and emphasized cultural and language ability.
The Healthy Schools Act supported the inclusion of mental health services in school health centers and procedures to ensure payment to school centers certified by the Department of Health and Human Services under Medicaid and the State Children's Health Insurance Program. In addition, the legislation recognizes the need to treat mental health as part of comprehensive health care, and to this end, it was necessary to establish a minimum standard for "primary health care services" as the core group of services provided by school health centres, including comprehensive health and mental health assessment, intervention and treatment [17].

In China, great importance is also attached to mental health education. In 2008, the National Health Commission released the Guideline on the Development of the National Mental Health System for 2008-2015 [18]. One of the priorities is to improve the coverage of mental health education at school. In 2010, the Ministry of Education again emphasized the importance of strengthening mental health education in the Outline of the National Medium and Long-term Educational Reform and Development Plan for 2010-2020 [19]. In accordance with national policy, Shanghai formulated a Three-year Action Plan for Mental Health Education in 2008 [20]. It served as a blueprint for establishing mental health education guidance centers for all the districts offering professional and standard training to psychological teachers. In addition, Shanghai also called on schools, families, communities, and medical institutions to participate in youth crisis prevention jointly. In 2012, Shanghai Mental Health Education Development Center was set up [21]. Its main functions and responsibilities include providing overall plans of mental health education and professional guidance, carrying out forward-looking research on mental health education, organizing and coordinating training for teachers, building a professional service network for students and establishing an international platform for cooperation and academic exchanges. All of these policies and practices promoted the development and implementation of mental health education in Shanghai.

\section{RESEARCH METHODS}

A previous study in China tested the effectiveness of an 8-week intensive mental health education course, namely the College Student Mental Health Education Course (CSMHEC) [22]. In September 2019, China Medical University provided CSMHEC to first-year medical students. This is a quasi-experimental study with both quantitative and qualitative analyses and recruited 374 first-year medical students as subjects with 188 (age $=17.97 \pm 0.65$ years, $37.2 \%$ male) in the experiment group and 186 (age $=18.02 \pm 0.63$ years, $40.3 \%$ male) in the control group. Medical students in the experiment group saw a significant decline in psychological distress $(\mathrm{p}<0.001, \mathrm{~d}=0.31)$ and academic burnout $(\mathrm{p}<0.001, \mathrm{~d}$ $=1.46)$, while they experienced a significant increase in 
life satisfaction levels after the intervention $(\mathrm{p}<0.001$, $\mathrm{d}$ $=0.48$ ). Compared with students in the control group, students in the experiment group had statistically significant lower levels of psychological distress $(\mathrm{p}<$ $0.05, \mathrm{~d}=0.23$ ) and academic burnout $(\mathrm{p}<0.001, \mathrm{~d}=$ 0.70 ), and statistically significant higher levels of life satisfaction in the post-test $(\mathrm{p}<0.01, \mathrm{~d}=0.31)$. Most students in the experiment group were satisfied with CSMHEC and themes extracted in the thematic analysis shed light on how the course could be improved. The mental health curriculum helped college students understand the features of their psychological development and related mental health problems such as stress, anxiety, depression, even self-suicide that may appear in their later lives. The literature inspired us to wonder if the mental health curriculum implemented in elementary and secondary schools could have the same effect. However, we found little literature on evaluating mental health education for elementary and secondary schools in China. Therefore, we carried out research to examine the effect of mental health programs on curbing adolescent suicide.

Since Shanghai formulated a Three-year Action Plan for Mental Health Education in 2008, it served as an ideal case for our research. We began to search for reports and studies on adolescent suicide in Shanghai and found data about adolescent suicide in Jiading District and Hongkou District in 2008 and 2012. We took the year 2008 as the control group and 2012 as the experimental group to form a natural experiment. We compare the number of suicides before and after the policy to explore the effectiveness of Shanghai mental health education.

\section{FINDINGS AND DISCUSSION}

We found the data of adolescent suicides in Jiading District and Hongkou District of Shanghai in 2008 and 2012, which is from a sample survey conducted by each district according to the survey model formulated by the Shanghai Municipal Center for Disease Control and Prevention (SCDC). Researchers used Chinese adolescent health-related behavior questionnaire and Shanghai adolescent health-related behavior questionnaire compiled by SCDC. The questionnaires include students' basic information, intentional suicidal behavior, etc. Suicidal behavior here was defined as: In the past 12 months, students had seriously considered suicide, and had planned to commit suicide, termed as a suicide plan, or had taken measures to attempt suicide, which was defined as attempted suicide. The sampling method is stratified multi-stage random sampling. The respondents were middle, high and vocational school students from some schools in Jiading and Hongkou District of Shanghai. The investigators had received professional training. With the help of the head teacher and unified guidance, each school employed anonymous questionnaire to assess students, with each class as a unit [23].
In 2008, investigators collected 727 valid questionnaires in Jiading District. The results showed that $80(11.2 \%)$ students in Jiading District had suicidal ideation in the past 12 months, $68(8.9 \%)$ had planned suicide and $28(3.7 \%)$ had attempted suicide. The rates of suicide planning and attempted suicide were higher than the average in Shanghai. Comparing suicide behavior of both genders and different school types, we can find that there are differences in suicide attempts among different schools $(\chi 2=14.593, p<0.05)$. The highest rate of suicide attempt was among vocational school students, followed by junior middle school students and the lowest among senior middle school students.

In 2012, the survey collected 1,089 valid questionnaires in the Jiading District of Shanghai [24]. The results showed that $117(10.8 \%)$ reported suicidal behavior in the past 12 months, of which $94(8.6 \%)$ had suicidal ideation, $50(4.6 \%)$ made suicide plans, and 23 $(2.1 \%)$ took suicide measures. When teenagers committed suicide, $76.9 \%$ did not seek help, $11.1 \%$ sought help from classmates and friends, 3.4\% sought help from others, $2.6 \%$ sought help from teachers, family members and net friends, while $0.9 \%$ sought help from doctors. The rate of taking suicide measures varied among different schools. The rate of suicide measures among middle school students was higher than that among high school students and vocational school students $(\chi 2=7.20, \mathrm{p}<0.05)$.

The survey used One-way Anova to analyze suicide behavior. The survey showed that grades, gender, parents' education background, father's education background, family structure would influence suicide behavior of middle school students. Girls are more likely than boys to commit suicide. Middle and high school students are more likely to commit suicide than vocational school students. Students with less educated parents are more likely to commit suicide. In terms of family structure, students from a nuclear family are more likely to commit suicide. Students with moderate academic performance are more likely to commit suicide, and students with good academic performance are less likely to commit suicide. On the premise of controlling other factors, sex is an important factor of suicidal behavior, and female students are 1.628 times more likely to commit suicide than male students.

In 2008, the Hongkou District conducted a questionnaire survey among 1,047 students [25]. The results showed that 139 (13.3\%) students in Hongkou had suicidal ideation in the past 12 months, $74(7.1 \%)$ had planned suicide, $32(3.1 \%)$ had attempted suicide and 11 $(1.1 \%)$ had committed multiple suicides. Similarly, there are also differences in suicidal ideation and attempted suicide among different school types. Middle and high school students are more likely to have suicidal ideation, middle school students and vocational school students 
are more likely to take suicide measures, and there is no significant difference in other aspects.

In 2012, the Hongkou District conducted a questionnaire survey among 1,413 middle school students [26]. The results showed that 316 students (22.43\%) had committed suicide, of whom 181 (12.88\%) had suicidal ideation, $100(7.08 \%)$ had suicide plan and $35(2.48 \%)$ had attempted suicide. There was significant difference between the two groups $(\chi 2=5.3256 .086, \mathrm{p}<$ $0.05)$. Among the students who had committed suicide, only $16.40 \%$ had sought help, of which $53.8 \%$ had sought help from classmates, $19.23 \%$ had sought help from parents, and only $9.62 \%$ had sought help from teachers.

According to those surveys, suicide rates among middle school students in the Hongkou and Jiading District have declined. We also find that the rate of suicide among middle school students in Hongkou District was higher than that in Jiading District.

\section{CONCLUSION}

Our study shows that mental health education does make contributions to reducing adolescent suicide. We also identify several reasons why mental health curriculum can help reduce adolescent suicide. First and foremost, diverse mental health programs enable students to have a thorough understanding of life and health, cultivate right attitudes towards suicide, and improve their problem-solving ability. They are less likely to collapse when faced with failure or difficulty.

What's more, social support is a protective factor in this regard. These school-based programs also integrate the role of peers and families, who have the closest relationship and direct contact with adolescents. With their company, adolescents can cope better with various problems, and enjoy better psychological and physical health. After receiving certain training, their friends and family members can sense any abnormality and deal with crisis as soon as possible. On the other hand, when troubled adolescents come to them for help or comfort, they can respond appropriately and report to professionals for further advice. Their support is an integral part of the whole project.

Another reason lies in its extensiveness and coordination. Schools can cooperate with medical institutions and counseling centers. For one thing, these organizations provide professional and comprehensive guidance for schools, like offering hotline, training staff, giving lectures, etc. Screening programs, gatekeeper training programs and crisis response teams can create a coordinated effort that identifies youth at risk. For another, schools serve as ideal research subjects. Psychologists can conduct studies at schools that succeed to mitigate suicide through mental health education, and share experience with other schools.
In conclusion, mental health education can benefit adolescents through joint efforts. The example of Shanghai proves its efficacy in reducing suicide.

Despite our findings, we cannot find more successive data on suicide rates due to limited resources. Furthermore, the example of Shanghai alone is not sufficient to examine how effective mental health education is under different circumstances. Future research can be done in more regions with more adequate data, so as to provide theoretical support and empirical evidence for implementing and perfecting mental health education. With more investment and research, we believe, mental health education can benefit adolescents to a larger extent.

\section{REFERENCES}

[1] "Suicide: one person dies every 40 seconds." World Health Organization, www.who.int/news/item/0909-2019-suicide-one-person-dies-every-40seconds. Accessed April 29th, 2021.

[2] Michael R Phillips, Xianyun Li, Yanping Zhang, Michael Eddleston. Suicide rates in China. The Lancet. 2002;359(9325): 2274-2275.

[3] "Suicide." World Health Organization, www.emro.who.int/healthtopics/suicide/feed/atom.html. Accessed April 29th, 2021.

[4] Patrick W. O'Carroll, Alan L. Berman, Ronald W. Maris, Eve K. Moscicki, Bryan L. Tanney, Morton M. Silverman. Beyond the Tower of Babel: A Nomenclature for Suicidology. Suicide and LifeThreatening Behavior. 1996;26(3): 237-252.

[5] “Adolescent Health." World Health Organization, www.who.int/health-topics/adolescenthealth/\#tab=tab_1. Accessed April 29th, 2021.

[6] Dwight Evans, Edna Foa, Raquel Gur, Herbert Hendin, Charles O'Brien, Daniel Romer, Martin Seligman, Timothy Walsh. Treating and Preventing Adolescent Mental Health Disorders: What We Know and What We Don't Know: A Research Agenda for Improving the Mental Health of Our Youth. Oxford University Press. 2005.

[7] Troister, Talia, Ronald R. Holden. Comparing Psychache, Depression, and Hopelessness in Their Associations with Suicidality: A Test of Shneidman's Theory of Suicide. Personality and Individual Differences. 2010;49(7): 689-693.

[8] Shi Xiangdong, Lu Jie, Liang Huining. Research Progress of Mental Health and Risk Behavior of Adolescent. Modern Preventive Medicine. 2009;36(7): 1324-1326. 
[9] Suma P Chand, John T. Chibnall, Stuart J. Slavin. Cognitive Behavioral Therapy for Maladaptive Perfectionism in Medical Students: A Preliminary Investigation. Academic Psychiatry. 2017;42(1): $58-61$.

[10] Katherine S. Hu, John T. Chibnall, Stuart J. Slavin. Maladaptive Perfectionism, Impostorism, and Cognitive Distortions: Threats to the Mental Health of Pre-clinical Medical Students. Academic Psychiatry. 2019;43(4): 381-385.

[11] "Withdrawal (defense mechanism)." Psychology Wiki,www.psychology.wikia.org/wiki/Withdrawal _(defense_mechanism). Accessed April 29th, 2021.

[12] Thomas Kötter, Josefin Wagner, Linda Brüheim, Edgar Voltmer. Perceived Medical School Stress of Undergraduate Medical Students Predicts Academic Performance: An Observational Study. BMC Medical Education. 2017;17(1): 256.

[13] Turley, B., \& Tanney, B. Evaluation Report on Suicide Intervention Field Trial Australia. Deakin, Australia: Lifeline Australia, Inc. and LivingWorks Education., Inc. 1998.

[14] Shaffer David, Garland Ann, Vieland Veronica, Underwood Maureen \& Busner Carey.The Impact of Curriculum-based Suicide Prevention Programs for Teenagers. 1991.

[15] Li Guoqiang, Li Fenglian. Characteristics and Enlightenment of Mental Health Education Policies in Foreign Schools. Journal of Hunan University of Humanities, Science and Technology. 2015;1:103106.

[16] Stephan, S. H., Weist, M., Kataoka, S., Adelsheim, S., \& Mills, C. Transformation of Children's Mental Health Services: The Role of School Mental Health. Psychiatric Services. 2007;58(10): 1330-1338.

[17] Ministry of Education of the People's Republic of China.

old.moe.gov.cn/publicfiles/business/htmlfiles/moe/ s3325/201212/145679.html. Accessed April 29th, 2021.

[18] National Health Commission of the People's Republic of China. www.nhc.gov.cn/zwgk/jdjd/201304/4836d140e040 49f988d3f968ee441c03.shtml. Accessed April 29th, 2021.

[19] Ministry of Education of the People's Republic of China. www.moe.gov.cn/srcsite/A01/s7048/201007/t2010 0729_171904.html. Accessed April 29th, 2021.
[20] China News.

www.chinanews.com/edu/zcdt/news/2008/1114/1449362.shtml. Accessed April 29th, 2021.

[21] Shanghai Municipal Education Commission. edu.sh.gov.cn/xxgk_jgzn_zsdw/20200514/0015gw_1052012003.html. Accessed April 29th, 2021.

[22] Qinghua Wang, Tianjiao Du. Implementation of the College Student Mental Health Education Course (CSMHEC) in Undergraduate Medical Curriculum: Effects and Insights. 2020;20: 505

[23] Zhang Yiying, Xu Lixin, Zhang Qin, Ma Feifei. Analysis of Health-related Risk Behaviors among Students in Secondary School in Jiading District. Health Education and Health Promotion. 2010;5(01):36-39.

[24] Zhang Qin, Dong Yuting, Zhang Fudong, Sun Sifei, Yuan Hong, Zhang Yiying. Characteristics and Enlightenment of Mental Health Education Policies in Foreign Schools. Chinese Journal of Health Education. 2015;(01):103-106.

[25] Huang Yang, Jiang Hua, Fan Hongen, Qi Deyun. Investigation and Analysis of Health Risk Behavior among the Youths in Hongkou District. Health Education and Health Promotion. 2009;4(01):11-15.

[26] Si Mei, Lin Ke, Qi Deyun, Fu Zejian, Li Xiangyun. Health Risk Behaviors among Adolescents in Hongkou District of Shanghai. Chinese Journal of School Health. 2014;07:986-988. 